\title{
Die Goldenen Zwanziger
}

Viele haben sich an der Schwelle zum neuen Jahrzehnt in der Prognose der langfristigen wirtschaftlichen Entwicklung versucht. Gefühlt überwiegen die Pessimisten, die sich einen Wettbewerb um die düsterste Prognose liefern. In spätestens fünf Jahren komme es zum größten Crash aller Zeiten: Demografie, Eurokrise, Auflösung der Währungsunion, Zinsen, Digitalisierung, Protektionismus, Krise der Automobilindustrie - alles scheint sich gegen die deutsche Volkswirtschaft zu wenden. Und schließlich demonstriert die weltweite Jugendbewegung Fridays for Future gegen den ökologischen Untergang. Interessant ist aber, wie zyklisch sich Narrative in Deutschland verändern: von der Lokomotive der 1990er über den kranken Mann der 2000er bis hin zum Superstar der 2010er. Soll es nun wieder zurück zum Sorgenkind gehen? Untergangsszenarien haben im Wirtschaftsdienst Tradition. Vor genau 100 Jahren lieferte der britische Ökonom und überzeugte Europäer John Maynard Keynes unter dem Eindruck der Verhandlungen über den Vertrag von Versailles einen bitteren Ausblick auf die 1920er: „Es mag uns daher ein langer, stiller Prozeß des Halbverhungerns und ein schrittweises, stetiges Sinken der Lebenshaltung bevorstehen.“

Natürlich wäre ein Crash bis spätestens 2025 keine Prognose, die sich mit hoher statistischer Wahrscheinlichkeit vorhersagen lässt. Die USA hatten immerhin seit der Finanzkrise über elf Jahre keine Rezession, was außergewöhnlich ist. Für die seriöse Prognose eines großen Crashs zu einem bestimmten Zeitpunkt reicht dies aber nicht. Zwar gibt es Annahmen über aktuelle Entwicklungen, aus denen sich Szenarien für die mittlere und lange Frist ableiten lassen. Darüber hinaus lassen sich diese Szenarien aber auch steuern, wenn sie mit geeigneten wirtschaftspolitischen Instrumenten beantwortet oder verhindert werden. Insofern können die potenziellen Krisen auch als wirtschaftspolitische Herausforderungen verstanden werden, die es anzunehmen gilt. Gesellschaftliche Sorgen und ökonomische Risiken weisen damit vor allem auf die Notwendigkeit wirtschaftspolitischer Gestaltung hin.

Die Herausforderungen, die sich aus der Digitalisierung sowie der Energie- und Verkehrswende ergeben, lassen sich mit Forderungen nach entsprechenden Investitionen beantworten. Investitionen in die digitale Infrastruktur und den Ausbau der erneuerbaren Energien sowie in die öffentliche Verkehrsinfrastruktur lösen die Probleme gestalterisch. Statt das Fliegen zu verbieten, könnte der öffentliche Verkehr ausgebaut werden. Es gibt eine Reihe von Umsetzungsfragen, insbesondere in Hinblick auf die Finanzierung, aber diese scheint angesichts der langanhaltenden Niedrigzinsphase zunehmend an Gewicht zu verlieren. Lange hat Deutschland auf Verschleiß gelebt und Investitionen unterlassen. Die schwarze Null konnte zwar die staatliche Bruttoschuldenquote um 20 Prozentpunkte reduzieren, eine Fortsetzung dieser Politik ließe die Schuldenstandsquote langfristig aber auf null sinken, was wenig sinnvoll ist, auch weil den Sparern sichere Anlagemöglichkeiten fehlen, und es keine Nachfrage nach Kredit gibt, was die Zinsen dämpft. Daher wird zunehmend infrage gestellt, ob ein weiterer Abbau der Staatsverschuldung angesichts der niedrigen Zinsen sinnvoll ist. Jedenfalls könnte eine vielfach geforderte begrenzte Nettokreditaufnahme für investive Zwecke eine geeignete Weiterentwicklung im Rahmen der Schuldenbremse sein, auch um langfristige Investitionen in die öffentliche Infrastruktur zu ermöglichen und die nötigen Kapazitäten wieder aufzubauen. Ein Übergang zur goldenen Regel der Finanzpolitik,

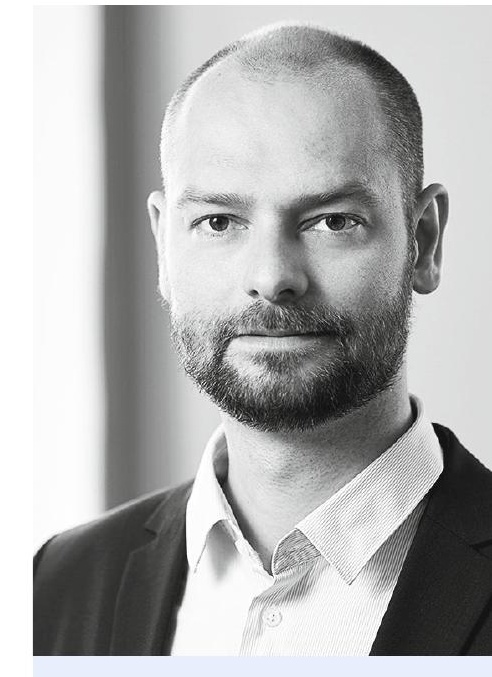

Christian Breuer ist

Chefredakteur von

Wirtschaftsdienst

und Intereconomics

in der ZBW - Leibniz-

Informationszentrum

Wirtschaft in Hamburg.

(C) Der/die Autor(en) 2020. Open Access: Dieser Artikel wird unter der Creative Commons Namensnennung 4.0 International Lizenz (https://creativecommons.org/licenses/by/4.0/deed.de) veröffentlicht.

Open Access wird durch die ZBW - Leibniz-Informationszentrum Wirtschaft gefördert. 
die Neuverschuldung für investive Projekte vorsieht, wäre gerade im Sinne der Generationengerechtigkeit, da den öffentlichen Schulden auch investiv geschaffene öffentliche Vermögen gegenüberstehen.

Höhere Investitionen sind auch geeignet, um Probleme der Europäischen Währungsunion zu adressieren. Sie erhöhen die Binnennachfrage in Deutschland, die die Sparschwemme absorbieren kann, und tragen dazu bei, Asymmetrien in Europa zu reduzieren. Ein Ausgleich der Leistungsbilanzen in Europa wäre zur Auflösung der Ungleichgewichte wünschenswert. Zudem sehen die europäischen Regeln - die Deutschland lange ignoriert hat - einen Ausgleich vor. Häufig wird die Erhöhung der Binnennachfrage in Deutschland aus konjunkturpolitischen Gründen abgelehnt. Deutschland sei überausgelastet und weitere Expansion würde Inflation erzeugen. Diese Schlussfolgerung hängt jedoch stark von den Annahmen ab. Zum einen hat die Inflation lange nicht die Zielinflation erreicht, was die Europäische Zentralbank (EZB) mit ihrer Niedrigzinspolitik zu beantworten versucht. Zum anderen sollte bei der Verteilung der Inflation innerhalb der Eurozone darauf geachtet werden, dass diese nicht nur aus Ländern mit geringer Wettbewerbsfähigkeit kommt, sondern aus Ländern, die niedrige Preisanpassungen hatten und deren Leistungsbilanzüberschüsse auf eine reale Unterbewertung hindeuten. Dazu gehören insbesondere Deutschland und die Niederlande. Die Sorge vor Inflation steht einer expansiven Politik in Deutschland also nicht entgegen. Sie wäre vielmehr die Voraussetzung für Zielinflation und höhere Zinsen.

Die demografische Entwicklung soll für langfristige wirtschaftliche Stagnation mitverantwortlich sein, bekannt als „säkulare Stagnation“. Paul Krugman sieht Europa in der Situation Japans vor 20 Jahren, mit einer chronischen Nachfrage- und Investitionsschwäche. Hinzu kommt eine Sparschwemme, die ebenfalls mit der demografischen Entwicklung verbunden wird. Aus beidem folgt ein niedriger Zins, der wiederum Ängste schürt, die sich um Vermögenspreise, Anlagevermögen und Inflation drehen. In der Tat zeigen Bevölkerungsprojektionen, dass in den kommenden 20 Jahren die Verrentung zunimmt. Aber auch die demografische Entwicklung kann gestaltet werden, z.B. durch Familien- und Migrationspolitik. Eine veränderte Politik zugunsten von Kindern und Familien wäre in der Lage, die Bevölkerungspyramide langfristig wieder auf die Füße zu stellen. Dies hätte eine erhebliche Nachfragewirkung. Darüber hinaus erhöht sich langfristig das Arbeitskräftepotenzial und verbessert damit eins zu eins die Situation der Rentenversicherung. Anders ist es, wenn die Geburten zurückgehen, wie seit den 1970er und 1980er Jahren. Aber dieser Trend ist keine Gesetzmäßigkeit. Eine Trendwende hat indessen bereits eingesetzt. Die Zukunft muss keine demografische Katastrophe werden und die Kräfte der säkularen Stagnation sind umkehrbar. Diese Szenarien verdeutlichen, dass die Zukunft in der langen Frist von Annahmen abhängig ist, die gestaltet werden können. Sie wird vor allem das sein, was wir daraus machen.

Auch für den Wirtschaftsdienst beginnt 2020 eine neue Zeit. Ab 1.1.2020 sind alle Beiträge Open Access, d.h., jeder erhält online kostenlos Zugriff auf Zeitschrift und Archiv. Zudem bietet sich mit der Open-Access-Lizenz (CC-BY) die Möglichkeit, die Beiträge uneingeschränkt weiter zu verbreiten und z.B. die Debatten zur Wirtschaftspolitik in sozialen Medien mit wissenschaftlichen Erkenntnissen zu bereichern. Ein freier Zugang verbessert so den Wissenstransfer zwischen der Forschung in den Wirtschaftswissenschaften. Die ZBW finanziert die Open-Access-Transformation. Für die Autorinnen und Autoren bzw. deren Institutionen entstehen dabei keine Kosten. Die beiden Zeitschriften Wirtschaftsdienst und Intereconomics erscheinen weiterhin beim Springer-Verlag.

Christian Breuer

ZBW - Leibniz-Informationszentrum

Wirtschaft

c.breuer@zbw.eu
Die Adressaten für wissenschaftliche Informationen sind auch Interessierte aus Medien, Politik und Zivilgesellschaft. Durch die Umstellung wird der Zugang zu wirtschaftspolitisch relevanten Erkenntnissen auch für nichtwissenschaftliche Leserinnen und Leser erheblich erleichtert. 